\title{
A modified decision tree approach to improve the prediction and mutation discovery for drug resistance in Mycobacterium tuberculosis
}

\author{
Wouter Deelder ${ }^{1,2}$, Gary Napier ${ }^{1}$, Susana Campino ${ }^{1}$, Luigi Palla ${ }^{1,3}$, Jody Phelan ${ }^{1 \dagger}$ and Taane G. Clark ${ }^{1,4^{*+}}$
}

\begin{abstract}
Background: Drug resistant Mycobacterium tuberculosis is complicating the effective treatment and control of tuberculosis disease (TB). With the adoption of whole genome sequencing as a diagnostic tool, machine learning approaches are being employed to predict M. tuberculosis resistance and identify underlying genetic mutations. However, machine learning approaches can overfit and fail to identify causal mutations if they are applied out of the box and not adapted to the disease-specific context. We introduce a machine learning approach that is customized to the TB setting, which extracts a library of genomic variants re-occurring across individual studies to improve genotypic profiling.

Results: We developed a customized decision tree approach, called Treesist-TB, that performs TB drug resistance prediction by extracting and evaluating genomic variants across multiple studies. The application of Treesist-TB to rifampicin (RIF), isoniazid (INH) and ethambutol (EMB) drugs, for which resistance mutations are known, demonstrated a level of predictive accuracy similar to the widely used TB-Profiler tool (Treesist-TB vs. TB-Profiler tool: RIF 97.5\% vs. 97.6\%; INH 96.8\% vs. 96.5\%; EMB 96.8\% vs. 95.8\%). Application of Treesist-TB to less understood second-line drugs of interest, ethionamide (ETH), cycloserine (CYS) and para-aminosalisylic acid (PAS), led to the identification of new variants $(52,6$ and 11 , respectively), with a high number absent from the TB-Profiler library $(45,4$, and 6 , respectively). Thereby, Treesist-TB had improved predictive sensitivity (Treesist-TB vs. TB-Profiler tool: PAS 64.3\% vs. 38.8\%; CYS 45.3\% vs. $30.7 \%$; ETH $72.1 \%$ vs. $71.1 \%$ ).

Conclusion: Our work reinforces the utility of machine learning for drug resistance prediction, while highlighting the need to customize approaches to the disease-specific context. Through applying a modified decision learning approach (Treesist-TB) across a range of anti-TB drugs, we identified plausible resistance-encoding genomic variants with high predictive ability, whilst potentially overcoming the overfitting challenges that can affect standard machine learning applications.
\end{abstract}

Keywords: Mycobacterium tuberculosis, Ethionamide, Cycloserine, PAS, Drug resistance, Machine learning

*Correspondence: Taane.clark@lshtm.ac.uk

†Jody Phelan and Taane G. Clark are Joint authors

${ }^{4}$ Department of Infection Biology, Faculty of Infectious and Tropical

Diseases, London School of Hygiene and Tropical Medicine, London, UK

Full list of author information is available at the end of the article

\section{Introduction}

Tuberculosis (TB), caused by Mycobacterium tuberculosis, is a pressing global health problem, with $>10$ million cases and 1.4 million associated deaths in 2019 [1]. First-line TB treatment uses combinations of the drugs rifampicin (RIF), isoniazid (INH), ethambutol (EMB) 
and pyrazinamide (PZA) [2]. Drug-resistance requires switching to second-line therapies combined in customized treatment protocols, which might include fluoroquinolones and injectable drugs, as well as ethionamide (ETH), cycloserine (CYS) and para-aminosalisylic acid (PAS), among others. Historically, a cascade of resistance has been defined, from resistance to RIF (RR-TB), to additional resistance to INH leading to multidrug resistance (MDR-TB), further leading to an extensively drug resistant (XDR-TB) class that is MDR-TB with additional resistance to fluoroquinolones and second-line injectables. Recently, there was a new definition of pre-XDR (MDR-TB and resistance to any fluoroquinolone) and an updated definition of XDR-TB (pre-XDR and resistance to least one additional Group A drug, including levofloxacin or moxifloxacin, bedaquiline and linezolid) [3]. These updates provide a framework for increasing progression of the severity of disease linked to resistance to additional anti-TB drugs [3].

The mechanisms that cause $M$. tuberculosis drug resistance are linked to genomic variants in drug targets or pro-drug activators, including single nucleotide polymorphisms (SNPs) and small insertions and deletions (indels), some occurring in gene-gene interactions. Prodrug activators convert mycobacterial enzymes that convert pro-drugs, such as INH and ETH, into their active form. If these enzymes (e.g., catalase peroxidase (KatG) for INH) are not essential, their coding genes can acquire mutations such as frameshifts which lead to loss of function, and consequently, the respective drug is not converted and resistance is caused. However, not all resistance mechanisms are well understood [4-6], especially for second-line drugs (e.g. PAS). Drug-resistance has been traditionally assessed through bacterial culture-based phenotypic drug susceptibility testing (DST), which can be time-consuming and resource intensive, with reproducibility and inhibitory concentration cutoff challenges for particular drugs [7]. Whole-genome sequencing (WGS) offers an alternative approach to infer resistance through the identification of associated genomic mutations [8], called "genotypic resistance" profiling. TB-Profiler software $[9,10]$ uses a curated library of $>1000$ mutations to predict genotypic resistance across 14 anti-TB drugs. The use of WGS can reaffirm known resistance mutations and uncover new candidates through genome-wide association studies (GWAS) and convergent evolution analysis [11]. However, GWAS approaches typically focus on single variants at a time in regression models, whereas resistance phenotype prediction from WGS is a classification problem with highdimensional input and potential complex interactions, a standard task in machine learning [12]. Therefore, the ongoing generation of large datasets using WGS is highly suited to the application of machine learning methods to improve "genotypic resistance" profiling [12].

The application of machine learning methods to $M$. tuberculosis has shown some impressive performances in genotypic profiling [13-17]. However, these models have several drawbacks that could affect their application in clinical settings, including their interpretability and an optimism bias related to the inclusion of non-associated cross-resistance and bacterial lineage markers; both leading to reduced predictive performance in hospital and other clinical settings [15]. The performance of machine learning models has also been relatively poor for a subset of second-line drugs (CYS, PAS, ETH), which in general are less often studied and analysed $[11,15]$. The generally lower performance for CYS, PAS and ETH suggests that mechanisms of resistance are less well understood, and that potentially rare alleles are being missed and excluded from models [15]. Our study aims to attempt to detect new genomic variants that might cause resistance for CYS, PAS, and ETH. The approach involves a customized (decision tree) machine learning algorithm, called Treesist-TB, which detects genomic variants in individual studies within the aggregated datasets, and can model variant interactions. It attempts to be robust to the presence of DST errors in some of the individual studies, which can lead to genomic variants being undetected in the analysis of the aggregate dataset.

\section{Results}

\section{Genomic and phenotypic data}

WGS data was available for 32,689 (32k) M. tuberculosis samples, which covered the main lineages 1 (9.6\%), 2 (25.2\%), 3 (11.4\%) and 4 (51.0\%) (S1 Table). Most samples were pan-susceptible (77.9\%), but RR-TB (1.3\%), MDR-TB (13.0\%) and XDR-TB (2.3\%) phenotypes were also represented. Phenotypic DST data was not available for all isolates, with limited data generation for PAS ( $n=1114,8.8 \%$ resistant), CYS ( $n=833,18.0 \%$ resistant), and ETH ( $n=2138,32.2 \%$ resistant) (S2 Table; Table 1$)$, as these drugs are mostly prescribed to and assessed in patients with RR-TB and MDR-TB.

\section{Application of Treesist-TB to first-line drugs}

Treesist-TB is a python-based machine learning algorithm that fits customized decision trees across individual studies and combines the extracted features to make final resistance predictions. It can also, if desired, be run assuming all data is from a single study (referred to as a "single optimised tree"). The algorithm was first applied to well-understood first-line drugs, using a subset of isolates that had complete DSTs (RIF: $n=2045,8.1 \%$ resistant, 7 studies; INH $n=1835,16.2 \%$ resistant; 6 studies; 
Table 1 Predictive performance across algorithms

\begin{tabular}{|c|c|c|c|c|c|c|c|c|c|c|}
\hline \multirow[t]{2}{*}{ Drug } & \multirow[t]{2}{*}{ Total tests } & \multirow[t]{2}{*}{$\%$ resistance } & \multicolumn{4}{|c|}{ TB-Profiler } & \multicolumn{4}{|c|}{ Treesist-TB $^{\mathrm{a}}$} \\
\hline & & & Sens & Spec & Acc & AUC & Sens & Spec & Acc & AUC \\
\hline $\mathrm{INH}$ & 1835 & 16.2 & 86.2 & 98.4 & 96.5 & 92.3 & 84.2 & 99.2 & 96.8 & 91.7 \\
\hline RIF & 2045 & 8.1 & 90.3 & 98.2 & 97.6 & 94.2 & 86.1 & 98.5 & 97.5 & 92.3 \\
\hline EMB & 1999 & 3.5 & 71.4 & 96.7 & 95.8 & 84.1 & 57.1 & 98.2 & 96.8 & 77.7 \\
\hline PAS & 1114 & 8.8 & 38.8 & 95.7 & 90.7 & 67.2 & 64.3 & 90.6 & 88.2 & 77.4 \\
\hline CYS & 833 & 18.0 & 30.7 & 95.2 & 83.6 & 62.9 & 45.3 & 93.7 & 85.0 & 69.5 \\
\hline \multirow[t]{3}{*}{ ETH } & 2118 & 32.2 & 71.1 & 78.6 & 76.2 & 74.8 & 72.1 & 75.8 & 74.6 & 73.9 \\
\hline & & & \multicolumn{4}{|c|}{ Regular Decision Tree } & \multicolumn{4}{|c|}{ Single optimized Tree ${ }^{b}$} \\
\hline & & & Sens & Spec & Acc & AUC & Sens & Spec & Acc & AUC \\
\hline $\mathrm{INH}$ & 1835 & 16.2 & 85.6 & 100 & 97.7 & 92.9 & 80.2 & 99.2 & 96.1 & 89.8 \\
\hline RIF & 2045 & 8.1 & 81.2 & 100 & 98.5 & 91.5 & 87.3 & 99.8 & 98.8 & 93.6 \\
\hline EMB & 1999 & 3.5 & 32.9 & 99.7 & 97.3 & 82.9 & 34.3 & 99.5 & 97.2 & 83 \\
\hline PAS & 1114 & 8.8 & 64.3 & 100 & 96.9 & 85.5 & 50 & 97.8 & 93.6 & 74.1 \\
\hline CYS & 833 & 18.0 & 33.3 & 99.4 & 87.5 & 67.3 & 35.3 & 98 & 86.7 & 66.7 \\
\hline ETH & 2118 & 32.2 & 48.8 & 94.3 & 79.7 & 77.5 & 49.6 & 92.5 & 78.7 & 76.2 \\
\hline
\end{tabular}

INH Isoniazid, RIF Rifampicin, PAS para-aminosalisylic acid, CYS cycloserine, ETH ethionamide, EMB Ethambutol, Sens Sensitivity, Spec Specificity, Acc Accuracy, AUC Area under the ROC Curve

a default application of Treesist-TB

${ }^{b}$ application of Treesist-TB with a single combined study dataset

EMB: $n=1999,3.5 \%$ resistant, 5 studies; S2 Table) across second-line drugs.

We fitted a default Treesist-TB tree assuming individual studies, as well as, for comparison purposes, single optimised and regular decision trees. The single optimized trees were simpler and contained fewer implausible sub-structures than regular decision trees (S2 Figure) while maintaining relevant structures such as double mutations and gene-gene interactions. In particular, the optimized trees contain fewer genes (INH: 27 vs. 5; RIF: 6 vs. 4; EMB: 5 vs. 4) but generally more individual variants (INH: 29 vs. 6; RIF: 15 vs. 20; EMB: 6 vs. 5) than regular decision trees. However, single optimized trees do include some unlikely features that might arise from overfitting on DST errors or other artefacts in the aggregated dataset (S2 Figure), so we applied the default Treesist-TB algorithm, which incorporates information from individual sub-studies.

The Treesist-TB algorithm identified several predictive genomic variants for resistance of RIF $(n=20 ; 7$ unreported in the TB-Profiler library), INH ( $n=20,13$ unreported) and EMB ( $n=10,2$ unreported) (S1 Figure, S2 Figure, Table 2; S4 Table). These included mutations in established loci such as $r p o B(n=18$, RIF), katG $(n=17$, $\mathrm{INH})$, and $e m b B(n=7, \mathrm{EMB})$. A confirmation analysis of the Treesist-TB mutations in the set of validation isolates ( $n=\sim 30 \mathrm{k}$ of $32 \mathrm{k}$, not analysed by Treesist-TB), revealed that none were present in susceptible strains, but they were frequent in both MDR-TB (median (maximum): RIF
1.6\% (65.3\%); INH <0.1\% (79.2\%); EMB $2.1(23.8))$ and XDR-TB (RIF 0.8\% (70.7\%); INH $<0.1 \%$ (78.6\%); EMB $3.1 \%(35.3 \%)$ ) isolates. The predictive accuracy of resistance from Treesist-TB was similar to the TB-Profiler tool (Treesist-TB vs. TB-Profiler: RIF $97.5 \%$ vs. $97.6 \%$; INH 96.8\% vs. $96.5 \%$; EMB $96.8 \%$ vs. $95.8 \%$ ), and like those from the single optimized and regular decision trees (Table 1), whose models include mutations not associated with resistance.

\section{Application to selected second-line drugs}

Given the strong performance for first-line drugs, Treesist-TB was then implemented for PAS, CYS and ETH, for which predictive accuracy has historically been poor and resistance mutations are only partially known [10]. Again, for comparison purposes, we fitted a single optimized tree for each drug and contrasted the performance and structure with regular classification trees (S3 Figure; Table 2). The results revealed that the optimized trees contain both fewer genes (PAS: 33 vs. 4; CYS: 7 vs. 3; ETH: 11 vs. 3) and variants (PAS: 37 vs. 7; CYS: 7 vs. 3; ETH: 13 vs. 5) than regular decision trees. The single optimized trees were simpler and contained fewer implausible sub-structures than regular decision trees, which appeared to be over-fitted (S2 Figure).

For PAS, the default application of Treesist-TB detected 11 genomic variants across three genes (folC 6, $R v 2670 c 1$, thyX 4) (Table 2). Six of the variants are unreported in TB-Profiler, occurring in folC (R49Q, Ser98G), 
Table 2 The Treesist-TB inferred variants

\begin{tabular}{|c|c|c|c|}
\hline Drug & Gene & $\begin{array}{l}\text { \# variants } \\
\text { in the } 32 \mathrm{k} \\
\text { dataset* }^{*}\end{array}$ & Treesist-TB Mutations** \\
\hline RIF & $r p o B$ & 757 & $\begin{array}{l}\text { N163K, V170F, L430P, Q432K, Q432L, D435Y, D435V, S441L, H445D, H445D, H445N, } \underline{\mathrm{H} 445 Y}, \mathrm{H445R}, \mathrm{H} 445 \mathrm{~L}, \underline{\mathrm{S} 450 \mathrm{~L}}, \\
\underline{\mathrm{L} 452 \mathrm{P}}, \mathrm{I} 91 \mathrm{~F}\end{array}$ \\
\hline RIF & rpoC & 700 & N1239D, Q1289A \\
\hline $\mathrm{INH}$ & $a h p C$ & 31 & $-57 \mathrm{C}>\mathrm{T},-48 \mathrm{G}>\mathrm{A}$ \\
\hline $\mathrm{INH}$ & fabG1 & 26 & $\underline{-126 G>A}$ \\
\hline $\mathrm{INH}$ & katG & 648 & $\begin{array}{l}\text { Y597D, T568P, A476V, S315T, S315N, S302R, W300C, G297V, P193fs, L159F, G156D, A144V, D142G, L141F, } \\
\text { N138D, A109V, Y98C }\end{array}$ \\
\hline EMB & embA & 743 & -31 delC, $-16 \mathrm{C}>\mathrm{T},-16 \mathrm{C}>\mathrm{A}$ \\
\hline EMB & $e m b B$ & 762 & 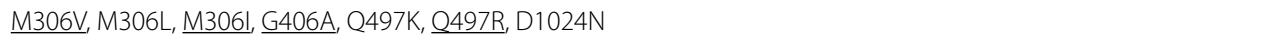 \\
\hline PAS & Rv2670c & 191 & $\underline{\mathrm{A} 5 \mathrm{~V}}$ \\
\hline PAS & foll & 262 & Q153G, Q153A, S150G, S98G, R49Q, 143T \\
\hline PAS & thy $X$ & 148 & $-4 \mathrm{C}>\mathrm{T},-\mathbf{9 G}>\mathrm{A},-16 \mathrm{C}>\mathrm{T},-\mathbf{1 8 G}>\mathrm{T}$ \\
\hline CYS & alr & 239 & Y388D, L283P, L113R, T20M \\
\hline CYS & rpoC & 700 & D485Y, I491T \\
\hline ETN & ethA & 494 & $\begin{array}{l}\text { W455*, K448fs, P436fs, A352fs, P334A, F320S, L295fs, C294*, R279*, Q269*, M260I, W256*, C253F, T236fs, } \\
\text { Y235fs, W228*, N226fs, K224*, A222V, S208L, R207G, V202F, L194P, T186P, P164R, P160fs, C137R, C137R, } \\
\text { W116*, K103fs, W45*, K37fs, L35R, Q24*, D6fs }\end{array}$ \\
\hline ETN & fabG1 & 26 & $-118 \mathrm{C}>\mathrm{G},-34 \mathrm{C}>\mathrm{T},-15 \mathrm{C}>\mathrm{T},-8 \mathrm{~T}>\mathrm{C},-8 \mathrm{~T}>\mathrm{A}$ \\
\hline ETN & gyrA & 764 & $\underline{\mathrm{A} 90 \mathrm{~V}}, \underline{\mathrm{S91P}}, \underline{\mathrm{D} 94 \mathrm{~A}}, \underline{\mathrm{D} 94 \mathrm{G}}$ \\
\hline ETN & $\operatorname{inh} A$ & 108 & 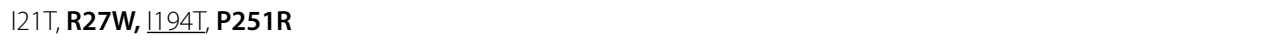 \\
\hline ETN & $m s h A$ & 250 & A133fs, H175fs, V237L, A422V \\
\hline
\end{tabular}

* $32 \mathrm{kM}$. tuberculosis isolates [18]

** Bolded if not in TB-Profiler in https://github.com/jodyphelan/tbdb/blob/master/tbdb.csv; * stop codon INH Isoniazid, RIF Rifampicin, PAS para-aminosalisylic acid, CYS cycloserine, ETH ethionamide, EMB Ethambutol

** Mutations underlined if they are in $>5 \%$ of MDR-TB or XDR-TB strains in the $32 \mathrm{kM}$. tuberculosis isolates

$R v 2670 c$ (A5V), and thy $X$ (three indels: $-4 \mathrm{C}>\mathrm{T},-9 \mathrm{G}>\mathrm{A}$, $-18 \mathrm{G}>\mathrm{T}$ ) (S5 Table). These PAS mutations were present in XDR-TB samples in the validation set (frequency: median 0.2\%, max. 6.1\%) (S5 Table). For PAS, compared to TB-Profiler, the Treesist-TB mutation set leads to a higher sensitivity (64.3\% vs. $38.8 \%)$, lower specificity $(90.6 \%$ vs. $95.7 \%)$ and similar overall accuracy $(88.2 \%$ vs. 90.7\%) for drug resistance prediction (Table 1). For CYS, Treesist-TB identified six variants across two genes (rpoC 2, 1 unreported; alr 4, 3 unreported). $R p o C$ is a locus linked to compensatory effects in RIF resistance. The CYS mutations were present in XDR-TB samples in the validation set (frequency: median $<0.1, \max .8 .5 \%$ ) (S5 Table). Compared to TB-Profiler, the set of TreesistTB mutations had a higher sensitivity ( $45.3 \%$ vs. $30.7 \%)$, and similar specificity ( $93.7 \%$ vs. $95.2 \%)$ and overall accuracy $(85.0 \%$ vs. $83.6 \%)$ for resistance prediction. For ETH, Treesist-TB identified 52 genomic variants, more than half in eth $A$ (35; 67.3\%), with others found across four genes (inhA 4, gyrA 4, mshA 4, fabG1 promoter 5). Most variants are not present in the TB-Profiler library (ethA 34, inhA 2, mshA 4, fabG1 promoter 5). EthA, fabG1 promoter and inhA are established ETH related loci, but
gyrA is linked to fluoroquinolone resistance, and $m s h A$ is known to encode a glycosyl-transferase enzyme involved in mycothiol biosynthesis that can affect ETH activation. These mutations for ETH were present in XDR-TB samples in the validation set (frequency: median $<0.1 \%$, max. 36.5\%) (S5 Table). For ETH, compared to TB-Profiler, Treesist-TB has a marginally higher sensitivity $(72.1 \%$ vs. $71.1 \%)$, lower specificity ( $75.8 \%$ vs. $78.6 \%)$ and a similar overall accuracy $(74.6 \%$ vs. $76.2 \%)$ for drug resistance prediction.

\section{Discussion}

The relatively poor knowledge of underlying mutations for second-line anti-TB drug resistance will make prospects for WGS-informed clinical and infection control more difficult. Whilst machine learning has the promise to fill any gaps in "genetic" knowledge, some implementations for M. tuberculosis "genotypic profiling" have led to over-optimistic predictive abilities and models with mutations that are not biologically plausible or unrelated to the resistance of interest. Our work describes a decision tree machine learning approach, called Treesist-TB, which attempts to account for inter-study differences 
and constrains the size of models, thereby minimising the risk of over-fitting due to phylogenetic or false resistance-associated mutations. Its application to RIF, INH and EMB drugs, with known resistance mechanisms, detected both established and unreported mutations in functional pathways, and had predictive abilities similar to other machine learning implementations and the TB-Profiler tool. Application of Treesist-TB to CYS, PAS and ETH drugs, whose underlying resistance variants are less established and are less often studied, detected putative non-synonymous SNPs and frameshift mutations in activation pathways. For the PAS drug, genomic variants were found in the folC gene, which interrupts bioactivation within the folate biosynthetic pathway [19]. Similarly, mutations were found in the alr gene encoding alanine racemase that compensates for the inhibitory effect of CYS [20]. Finally, for ETH, the majority of mutations were detected in the eth $A$ gene that activates $\mathrm{ETH}$ by the NADPH-specific flavin adenine dinucleotide-containing monooxygenase EthA [21]. Importantly, integrated WGS and DST studies for relatively new antiTB drugs (e.g., bedaquiline, clofazimine and delamanid) are much-needed, as current low sample sizes make the determination of mutations underlying their resistance difficult [22].

Treesist-TB detects SNPs by working with the largest datasets possible, where some of the reported performance problems for second-line drugs are due to the exclusion of rare alleles. More importantly, Treesist-TB considers individual sub-studies that make up the large dataset, implicitly adjusting for potential DST or mislabelling errors in individual studies, which are potentially more common in some laboratories or drug assays. Treesist-TB also incorporates existing knowledge on which sub-structures in the decision trees are biologically less plausible, such as reversion mutations, and can prune these structures. If required, the approach can give preference to known resistance genomic variants in tree model building and control its complexity by placing a ceiling on the number of previously unknown resistance mutations. In this sense, Treesist-TB can take advantage of prior knowledge and insights specific to TB drug resistance, thereby providing a counterweight against the increasing usage of machine learning "out of the box", which can lead to models that do not generalize well in clinical practice.

Our analysis revealed that standard machine learning approaches could, even after regular cross-validation, overfit in subtle manners that lead to an upward bias in performance and not translate into a high out-of-training-set performance. Although, a robust simulation study that considers a number of machine learning approaches is beyond the scope of our work, previous studies have shown that some implementations have boosted performance through the selection of cross-resistance markers that are unlikely to be causally related to resistance to the drug under investigation [15]. These unrelated markers might get selected as features by machine learning models due to the unique structure of TB datasets, including arising from $M$. tuberculosis phylogenetic structures and sequential drug testing practices. Similarly, fitted tree structures with features that are biologically unrelated to resistance might lead to impressive performance within the training set, but may be inappropriate for predictions in clinical practice. These problems will be exacerbated for more complex models that have a greater number of parameters, such as convolutional neural nets [23].

\section{Conclusions}

In general, with the increasing application of WGS data in a clinical or research setting, there is a need for robust and interpretable machine learning models that take advantage of the resulting large and growing datasets, whilst being robust to data errors. One important application is in antimicrobial resistance (AMR) genotypic profiling, which could ultimately replace phenotypic DST approaches. However, any AMR models derived must be reliable in terms of prediction, generalize across clinical settings, and adapt to increasing data and knowledge. In addition, such models need to account for the idiosyncrasies of pathogens and infections, where $M$. tuberculosis is highly clonal and has no horizontal gene transfer, but for other pathogens there may be plasmid derived AMR. In conclusion, we have developed Treesist-TB, which can assist with identifying mutations and prediction drug resistance in a TB context. Through providing software for its implementation, the utility beyond TB can be evaluated, and the approach potentially refined for other AMR settings.

\section{Materials and methods Phenotypic and sequencing data}

The main dataset consists of 32,689 (32k) isolates with whole genome sequencing (WGS) and phenotypic drug susceptibility test (DST) data (see S1 Table [18];). The laboratory DST followed WHO recommended protocols and practice (see [11]). XDR-TB was defined using the recently replaced definition, that is, being MDR-TB with additional resistance to fluoroquinolones and second-line injectables. This is because the isolates were collected, processed, and resistance patterns interpreted for treatment options before the new definitions were introduced [3]. DST data was not available for every isolate across all drugs, as only those individuals resistant to first-line treatments are typically tested for second-line resistance. All isolates with PAS, CYS 
and ETH DST were included in the analysis (see S2 Table for sample sizes). A subset with complete INH, RIF and EMB DST data and with similar characteristics in terms of sample size and number of individual studies were chosen for Treesist-TB benchmark analysis (S2 Table). The residual $31 \mathrm{k}$ isolates were used for validation through the analysis of mutation frequencies across susceptible and resistance groups. The raw sequence data were mapped to the $\mathrm{H} 3 \mathrm{Rv}$ reference genome using bwa-mem software, and genomic variants (SNPs, indels) were called from the consensus of GATK and samtools software. Most genomic variants (98.9\%) have low minor allele frequencies $(<1 \%)$, and we excluded SNPs in hypervariable PE/PPE gene families and with synonymous mutations (see [18]).

\section{Treesist-TB model}

The Treesist-TB model is a major extension of a simple decision tree approach (sklearn implementation, v0.23.1) with the following modifications: (1) incorporation of prior parameters on which features to prioritize in the tree building in case of ties; 2) incorporation of tree pruning to limit interactions in the tree that are $a$ priori determined to be unlikely (e.g. double mutations that compensate resistant mutations and restore drug sensitivity); (3) incorporation of prior parameters for the maximum number of genes (not genomic variants) in a tree. Although Treesist-TB is compatible with regular cross-validation methods (e.g., leave k-fold out), these approaches may lead to unstable results for trees in general. To prevent trees from having excessive depth, the setting of priors for the maximum number of new genes outside known resistance genes (not variants) has been implemented. We extracted a set of genomic variants using a consensus rule that variants were only included when in genes that were more than once detected across sub-datasets (S1 Figure).

\section{Model fitting}

The predictive performance of the final models fitted to the entire dataset was measured using sensitivity, specificity, accuracy, and area under the ROC curve (AUC) metrics, assuming DST results as the gold standard. We compared the performance of the (default) TreesistTB model primarily with the TB-Profiler software and mutation library (>1000 SNPs, indels or large deletions) $[9,10]$. In addition, for comparison, we fitted a regular decision tree model and Treesist-TB (labelled as "Single optimized Tree") on aggregate datasets. The depth of the regular decision tree was set by 5 -fold cross-validation up to a maximum of 15 .

\section{Packages}

The pipeline was implemented in Python (v3.7), building on the tree algorithm from sklearn (v0.23.1). The plausibility of putatively causal genomic variants identified was assessed using Mycobrowser [24].

\section{Supplementary Information}

The online version contains supplementary material available at https://doi. org/10.1186/s12864-022-08291-4.

Additional file 1.

\section{Acknowledgements}

We thank Aleksei Ponomarev and Gabriel Marzinotto for coding support and public code that was used in the development of Treesist-TB.

\section{Authors' contributions}

WD, JP and TGC conceived and designed the study. GN and JP performed the bioinformatic processing of the raw sequencing data and phenotypic data; WD developed the algorithm and performed the statistical analysis, under supervision of SC, LP, JP, and TGC. WD wrote the first draft of the manuscript, and the final version included edits from all authors. The final manuscript was read and approved by all authors.

\section{Funding}

JP is funded by a Newton Institutional Links Grant (British Council, no. 261868591). TGC is funded by the Medical Research Council UK (Grant no. MR/ M01360X/1, MR/N010469/1, MR/R025576/1, and MR/R020973/1) and BBSRC (Grant no. BB/R013063/1). SC is funded by Medical Research Council UK grants (ref. MR/M01360X/1, MR/R025576/1, and MR/R020973/1).

\section{Availability of data and materials}

The raw whole genome sequencing data is available from the European Nucleotide Archive (ENA) (see [18]). Computing code is available at https:// github.com/WDee/Treesist-TB.

\section{Declarations}

Ethics approval and consent to participate

Not applicable.

Consent for publication

All authors approve the publication.

\section{Competing interests}

There are no competing interests. WD was employed by the company Dalberg Advisors in Switzerland. The remaining authors declare that the research was conducted in the absence of any commercial or financial relationships that could be construed as a potential conflict of interest.

\section{Author details}

${ }^{1}$ London School of Hygiene \& Tropical Medicine, Keppel Street, London WC1E 7HT, UK. ${ }^{2}$ Dalberg Advisors, 7 Rue de Chantepoulet, CH-1201 Geneva, Switzerland. ${ }^{3}$ Department of Public Health and Infectious Diseases, University of Rome La Sapienza, Rome, Italy. ${ }^{4}$ Department of Infection Biology, Faculty of Infectious and Tropical Diseases, London School of Hygiene and Tropical Medicine, London, UK.

Received: 25 June 2021 Accepted: 3 January 2022

Published online: 11 January 2022

\section{References}

1. World Health Organization. Tuberculosis Factsheet 2018.

2. World Health Organization. DS TB Treatment Factsheet 2017. 
3. World Health Organization. Meeting report of the WHO expert consultation on drug-resistant tuberculosis treatment outcome definitions, 17-19 November 2020. In: World Health Organization [Internet]. 2020 p. 14. Available: https://apps.who.int/iris/handle/10665/340284

4. Trauner A, Borrell S, Reither K, Gagneux S. Evolution of drug resistance in tuberculosis: recent progress and implications for diagnosis and therapy. Drugs. 2014;74:1063-72. https://doi.org/10.1007/s40265-014-0248-y.

5. Safi H, Lingaraju S, Amin A, Kim S, Jones M, Holmes M, et al. Evolution of high-level ethambutol-resistant tuberculosis through interacting mutations in decaprenylphosphoryl- $\beta$-D-arabinose biosynthetic and utilization pathway genes. Nat Genet. 2013;45:1190-7. https://doi.org/10.1038/ng. 2743.

6. Gygli SM, Borrell S, Trauner A, Gagneux S. Antimicrobial resistance in mycobacterium tuberculosis: mechanistic and evolutionary perspectives. FEMS Microbiol Rev. 2017;41:354-73. https://doi.org/10.1093/femsre/ fux011.

7. Farhat MR, Sultana R, lartchouk O, Bozeman S, Galagan J, Sisk P, et al. Genetic determinants of drug resistance in mycobacterium tuberculosis and their diagnostic value. Am J Respir Crit Care Med. 2016;194:621-30. https://doi.org/10.1164/rccm.201510-20910C.

8. Dheda K, Gumbo T, Maartens G, Dooley KE, McNerney R, Murray M, et al. The epidemiology, pathogenesis, transmission, diagnosis, and management of multidrug-resistant, extensively drug-resistant, and incurable tuberculosis. Lancet Respir Med. 2017;5:291-360. https://doi.org/10.1016/ S2213-2600(17)30079-6.

9. Coll F, McNerney R, Preston MD, Guerra-Assunção JA, Warry A, Hill-Cawthorne $\mathrm{G}$, et al. Rapid determination of anti-tuberculosis drug resistance from whole-genome sequences. Genome Med. 2015;7:51. https://doi. org/10.1186/s13073-015-0164-0.

10. Phelan JE, O'Sullivan DM, Machado D, Ramos J, Oppong YEA, Campino S, et al. Integrating informatics tools and portable sequencing technology for rapid detection of resistance to anti-tuberculous drugs. Genome Med. 2019;11:41. https://doi.org/10.1186/s13073-019-0650-x.

11. Coll F, Phelan J, Hill-Cawthorne GA, Nair MB, Mallard K, Ali S, et al. Genome-wide analysis of multi- and extensively drug-resistant mycobacterium tuberculosis. Nat Genet. 2018;50:307-16. https://doi.org/10.1038/ s41588-017-0029-0

12. Libiseller-Egger J, Phelan J, Campino S, Mohareb F, Clark TG. Robust detection of point mutations involved in multidrug-resistant mycobacterium tuberculosis in the presence of co-occurrent resistance markers. PLoS Comput Biol. 2020;16. https://doi.org/10.1371/journal.pcbi.1008518.

13. Kouchaki S, Yang Y, Walker TM, Walker AS, Wilson DJ, Peto TEA, et al. Application of machine learning techniques to tuberculosis drug resistance analysis. Wren J, editor. Bioinformatics. 2018. https://doi.org/10.1093/bioin formatics/bty949.

14. Yang Y, Niehaus KE, Walker TM, lqbal Z, Walker AS, Wilson DJ, et al. Machine learning for classifying tuberculosis drug-resistance from DNA sequencing data. Bioinformatics. 2018;34:1666-71. https://doi.org/10. 1093/bioinformatics/btx801.

15. Deelder W, Christakoudi S, Phelan J, Benavente ED, Campino S, McNerney $\mathrm{R}$, et al. Machine learning predicts accurately mycobacterium tuberculosis drug resistance from whole genome sequencing data. Front Genet. 2019:10. https://doi.org/10.3389/fgene.2019.00922.

16. Yang Y, Walker TM, Walker AS, Wilson DJ, Peto TEA, Crook DW, et al. DeepAMR for predicting co-occurrent resistance of mycobacterium tuberculosis. Bioinformatics. 2019. https://doi.org/10.1093/bioinforma tics/btz067.

17. Chen ML, Doddi A, Royer J, Freschi L, Schito M, Ezewudo M, et al. Deep learning predicts tuberculosis drug resistance status from whole-genome sequencing data. bioRxiv. 2018:275628. https://doi.org/10.1101/275628.

18. Napier G, Campino S, Merid Y, Abebe M, Woldeamanuel Y, Aseffa A, et al. Robust barcoding and identification of mycobacterium tuberculosis lineages for epidemiological and clinical studies. Genome Med. 2020;12:114. https://doi.org/10.1186/s13073-020-00817-3.

19. Minato Y, Thiede JM, Kordus SL, McKlveen EJ, Turman BJ, Baughn AD. Mycobacterium tuberculosis folate metabolism and the mechanistic basis for Para-aminosalicylic acid susceptibility and resistance. Antimicrobial agents and chemotherapy. American society for. Microbiology. 2015:5097-106. https://doi.org/10.1128/AAC.00647-15.

20. Chen J, Zhang S, Cui P, Shi W, Zhang W, Zhang Y. Identification of novel mutations associated with cycloserine resistance in mycobacterium tuberculosis. J Antimicrob Chemother. 2017;72:3272-6. https://doi.org/ 10.1093/jac/dkx316.

21. Vilchèze $C, W R J J R$. Resistance to isoniazid and Ethionamide in mycobacterium tuberculosis: genes, Mutations, and Causalities. Microbiol Spectr. 2014;2. https://doi.org/10.1128/microbiolspec.mgm2-0014-2013.

22. Gómez-González PJ, Perdigao J, Gomes P, Puyen ZM, Santos-Lazaro D, Napier G, et al. Genetic diversity of candidate loci linked to mycobacterium tuberculosis resistance to bedaquiline, delamanid and pretomanid. Sci Rep. 2021;11. https://doi.org/10.1038/s41598-021-98862-4.

23. Hastie T, Tibshirani R, Friedman J. The elements of statistical learning. New York: Springer New York; 2009. https://doi.org/10.1007/ 978-0-387-84858-7.

24. Kapopoulou A, Lew JM, Cole ST. The MycoBrowser portal: a comprehensive and manually annotated resource for mycobacterial genomes. Tuberculosis. 2011;91:8-13. https://doi.org/10.1016/J.TUBE.2010.09.006.

\section{Publisher's Note}

Springer Nature remains neutral with regard to jurisdictional claims in published maps and institutional affiliations.
Ready to submit your research? Choose BMC and benefit from:

- fast, convenient online submission

- thorough peer review by experienced researchers in your field

- rapid publication on acceptance

- support for research data, including large and complex data types

- gold Open Access which fosters wider collaboration and increased citations

- maximum visibility for your research: over $100 \mathrm{M}$ website views per year

At BMC, research is always in progress.

Learn more biomedcentral.com/submissions 